\title{
Economia da saúde
}

Health economics

Camila Pepe Ribeiro de Souza ${ }^{1}$ (D)

'Origin Health Company, São Paulo, SP, Brasil.

Autor correspondente: Camila Pepe Ribeiro de Souza Rua Sansão Alves dos Santos, $102,3^{\circ}$ andar CEP. 04571-090 - Cidade Monções E-mail: camila.pepe@originhealth.com.br

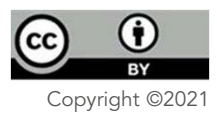

Nos últimos anos, a incorporação de novas tecnologias é parte do aumento dos custos em saúde no Brasil e no restante do mundo. ${ }^{1}$ Todos os recursos para prevenção, diagnóstico e tratamento de enfermidades, incluindo medicamentos, materiais médicos, equipamentos, procedimentos, bem como programas e protocolos assistenciais, além dos sistemas organizacionais, educacionais, de informação e de suporte, fazem parte das novas tecnologias. ${ }^{2}$ Anteriormente, os critérios do processo de incorporação para o reembolso de novas tecnologias eram exclusivamente de eficácia e segurança. No entanto, a definição de critérios econômicos é cada vez mais necessária, devido ao aumento de custos e de restrições orçamentárias dos sistemas de saúde. A análise dos critérios econômicos que subsidiam a tomada de decisão sobre a incorporação racional de novas tecnologias chama-se Avaliação de Tecnologias em Saúde (ATS).

A ATS determina o impacto clínico e econômico da incorporação de uma nova tecnologia, considerando-se a perspectiva de uma fonte pagadora específica. A primeira etapa dessas avaliações envolve a busca por estudos clínicos com análises das tecnologias em relação à eficácia e à segurança em bases de dados da área médica. O início dessa etapa ocorre a partir de uma pergunta central e se constitui de cinco componentes: doença, intervenção, desfecho clínico, grupo controle e tipo de estudo. Deve refletir o objetivo principal da pesquisa. Após essa etapa inicial de comprovação de ganhos clínicos da nova tecnologia em relação à tecnologia padrão, as etapas subsequentes são a quantificação de recursos e a comparação entre os custos das tecnologias. Os produtos que comprovadamente agregam valor clínico em relação à sua alternativa com menor custo global, por definição, são mais eficientes e devem ser implementados. No entanto, a maioria das novas tecnologias também está associada a maior custo.

As avaliações econômicas de custo-efetividade e custo-utilidade analisam os desfechos clínicos e o impacto econômico da incorporação da nova tecnologia em comparação à conduta padrão. Dessa forma, é possível avaliar se o retorno clínico justifica o investimento adicional em uma nova tecnologia.

Os desfechos das análises de custo-efetividade são os eventos clínicos e a sobrevida dos pacientes. As projeções para a comparação da custo-efetividade de uma nova tecnologia, quando se considera que a nova tecnologia prolonga a vida dos pacientes, por exemplo, incluem a sobrevida média esperada e os custos totais para os pacientes tratados com a nova tecnologia e a tecnologia padrão. Os custos abrangem tratamento farmacológico, acompanhamento por meio da realização de exames e consultas médicas e tratamento após a progressão da doença ou em caso de ocorrência de evento clínico desfavorável.

Após os cálculos das médias de expectativa de vida dos pacientes e dos custos totais, devem-se calcular os valores incrementais de sobrevida e de custo médio 
associados à cada alternativa. Dessa forma, avalia-se não só o quanto a nova tecnologia se mostra melhor do que a tecnologia padrão em termos clínicos, mas também o quanto seria mais cara do que a nova tecnologia.

Com esses dados, calcula-se a razão de custo-efetividade incremental (RCEI), principal resultado das avaliações econômicas. A RCEI é calculada pela divisão entre o custo incremental e a efetividade incremental. O resultado indica o quanto se gastaria a mais para se obter 1 ano de vida adicional com o uso da nova tecnologia, quando comparada à tecnologia padrão. Se os desfechos são eventos clínicos evitados, a RCEI representa o quanto se gastaria a mais para se evitar um evento clínico. A fórmula para cálculo da RCEI é a seguinte:

$$
\text { RCEI }=\frac{\text { Custo }_{\text {Nova tecnologia }}-\text { Custo }_{\text {Tecnologia padrão }}}{\text { Efetividade }_{\text {Nova tecnologia }}-\text { Efetividade }_{\text {Tecnologia padrão }}}
$$

A RCEI deve ser comparada com o limite de disposição a pagar dos gestores ou da sociedade como um todo por um ano de vida adicional para os pacientes. Não existe no Brasil um limite oficial de quanto se estaria disposto a pagar para salvar um ano de vida de um paciente. Quando uma nova tecnologia apresenta uma RCEI abaixo do limite de disposição a pagar do país em questão diz-se que a tecnologia é custo-efetiva quando comparada à tecnologia padrão.

Já na análise de custo-utilidade, são considerados os custos diretos e indiretos, e os desfechos são avaliados como anos de vida ajustados pela qualidade (QALYs, do inglês quality-adjusted life years). Os QALYs são compostos pela duração da sobrevida e pelo fator de qualidade de vida, também chamado de utilidade, ou "utility". O valor do QALY é calculado pela multiplicação dos anos de vida vividos pelo fator de utilidade associado ao estado de saúde dos pacientes. Os valores de utilidade podem variar de zero a um, sendo que zero representa a morte e um representa saúde perfeita. Um valor de utilidade de o,7, por exemplo, representa um estado de saúde com 70\% de qualidade de vida do estado de saúde perfeita.

O envelhecimento populacional, com consequente aumento da prevalência de doenças crônicas, e o surgimento de novas tecnologias implicam em alocação mais racional dos recursos financeiros, o que torna essenciais as avaliações econômicas pelos gestores em saúde.

A análise das condições clínicas impactantes e com mais necessidades não atendidas e o potencial de ganho com a incorporação de novas tecnologias para a população coberta por uma determinada operadora de planos de saúde são fatores que devem ser identificados e avaliados por cada gestor. Após esse mapeamento, as novas tecnologias potenciais devem ser submetidas à avaliação clínica e econômica, para a tomada de decisão racional sobre a alocação ótima dos recursos financeiros escassos.

\section{REFERÊNCIAS}

1. Vieira FS. Evolução do gasto com medicamentos do Sistema Único de Saúde no período de 2010 a 2016 . Instituto de Pesquisa Econômica Aplicada - IPEA; 2018. Disponível em: https://www.ipea.gov.br/portal/images/stories/PDFs/TDs/180117_td_2356.pdf

2. Brasil. Ministério da Saúde. Gabinete do Ministro. Portaria N².510/GM de 19 de dezembro de 2005. Institui Comissão para Elaboração da Política de Gestão Tecnológica no âmbito do Sistema Único da Saúde - CPGT. Brasília, DF: Ministério da Saúde; 2005. [citado 2021 Abr 16]. Disponível em: http://bvsms.saude. gov.br/bvs/saudelegis/gm/2005/prt2510_19_12_2005.html 